\title{
Merely miR210 in mesenchymal stem cells-one size fits all
}

\author{
Friedrich C. Luft
}

Published online: 13 July 2012

(C) Springer-Verlag 2012

MicroRNAs (miRs) are endogenous, single-stranded, noncoding RNAs about 18-24 nucleotides in length. miRs are highly conserved and ubiquitously expressed. They function as endogenous repressors of target genes by inhibiting translation, promoting degradation of mRNA, or increasing translation, in case they are turned off. Each miR can evidently influence hundreds of gene transcripts to coordinate gene expression programs. In the heart, miRs regulate cardiomyocyte hypertrophy and myocardial contractile function and modulate ischemia-reperfusion-induced apoptosis, fibrosis, angiogenesis, and remodeling. In other words, they do most everything any cardiologist would want. Their role in the heart, particularly the heart subjected to ischemia, has recently been reviewed [1].

Since much heart disease is somehow caused by ischemia, the physiological sensors of hypoxia, namely hypoxiainducible transcription factors (HIFs), have garnered deserved attention. Aside from erythropoietin, vascular endothelial growth factor, and many other obvious targets, HIF also regulates specific hypoxia-inducible miR, also termed "hypoxamirs." The number of hypoxamirs is already too numerous to count; suffice it to say that hypoxamir, miR210, appears to rage above the rest [2]. The stem-loop of miR-210 is located in an intron of a long noncoding RNA, which is transcribed from AK123483 on chromosome 11p. HIF $1 \alpha$ directly binds to a hypoxia responsive element on

F. C. Luft $(\bowtie)$

Experimental and Clinical Research Center,

Charité Medical Faculty and Max-Delbrück

Center for Molecular Medicine,

Lindenbergerweg 80 ,

13125 Berlin, Germany

e-mail: luft@charite.de the proximal miR-210 promoter. What does miR210 do? The answer is: "most everything." miR-210 influences cell growth arrest, supports stem cell survival, regulates mitochondrial metabolism, stalls DNA repair, induces angiogenesis, and supports cell differentiation [3]. Were there a versatile miR-like drug, an miR-210-related product would be it (Fig. 1).

Our parallel topic is mesenchymal stromal/stem cells (MSCs). The existence of precursor cells within mesenchyme has been known since the 1920s. Much more recent experimentation revealed the plasticity of bone marrow cells and how their fate could be determined by environmental cues. Culturing marrow stromal cells in the presence of osteogenic stimuli such as ascorbic acid, inorganic phosphate, and dexamethasone promotes their differentiation into osteoblasts. In contrast, the addition of transforming growth factor-beta induces chondrogenic markers. In 2012, a PubMed perusal identified over 17,000 references for MSC. However, thanks to a recent review, you do not need to read them all [4]. Characterization and documentation are pivotal. The minimum criteria for MSCs included plastic adherence and in vitro trilineage differentiation to adipogenic, chondrogenic, and osteogenic cells. Additional requirements included cell surface expression of CD105 (endoglin, SH2), CD73 (ecto-50-nucleotidase), and CD90 (Thy1) and the absence of the hematopoietic markers, CD45, CD19, CD19 or CD79, CD14 or CD11b, and HLA-DR. A particular challenge for the field has been the absence of a specific marker to define MSCs, although a large number of different determinants have been associated, albeit not exclusively, including CD271 (low-affinity nerve growth factor receptor) and CD146. In addition to bone marrow, MSC populations (Fig. 2) can be obtained 


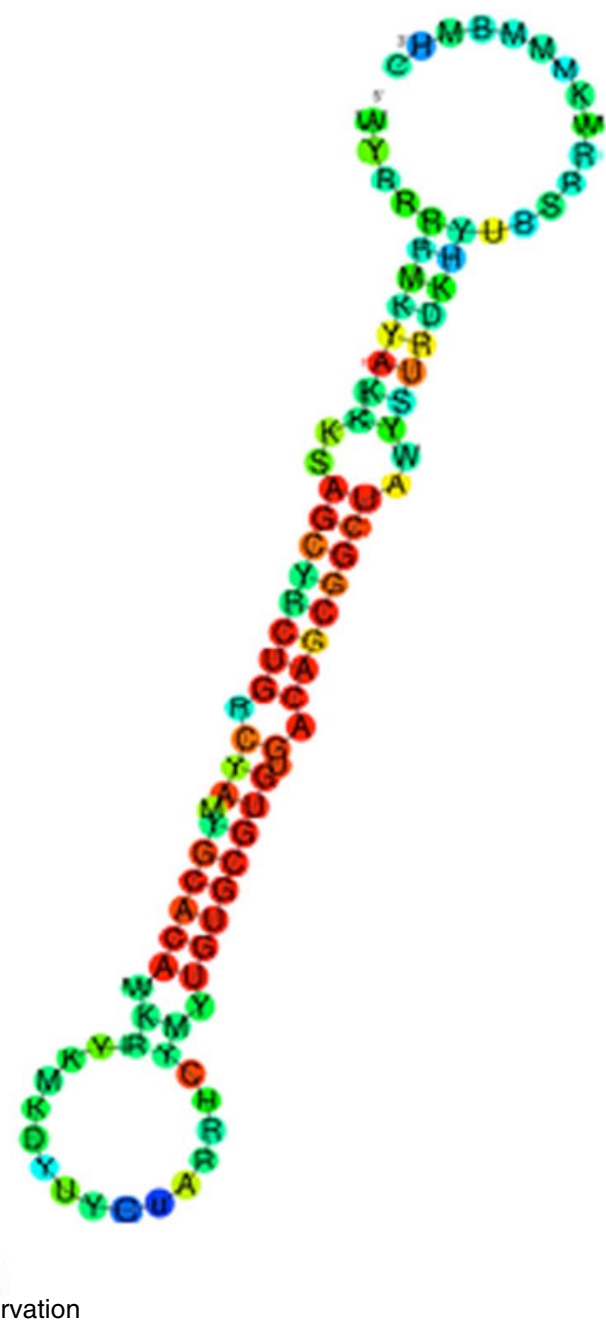

Sequence conservation

\section{Conserved secondary structure of mir-210}

Fig. 1 Conserved secondary structure of mir-210

Fig. 2 Mesenchymal stem cell pathway readily from adipose tissue, placenta, skin, umbilical cord blood, and umbilical cord perivascular cells [4]. MSCs have already found their way into the clinical arena. Since MSCs have remarkable immune-modulatory functions, including $\mathrm{T}$ cell suppression, a study using these cells in patients receiving kidney transplants has been published [5]. Three preliminary trials of MSC, derived from subsequent recipients, in heart disease are in the literature [6-8].

Kim et al. [9] now bring these two topics, namely miR and MSC, together [9]. First, the authors obtained MSC from bone marrow cavities of Fischer 344 rats. Plasmids containing miR-210 and scrambled RNA plasmids were then prepared. Polyethyleneimine nanoparticles were incubated with the plasmids in media $\left(\right.$ Opti-MEM $\left.{ }^{\circledR}\right)$. These complexes were added to the cells and incubated. The authors could then detect miR-210 with qRT-PCR or fluorescent in situ hybridization, to see if transfection was appropriate. The miR-210 transfections seemed to work. The authors then exposed their MSC to severe hypoxia, and by TUNEL staining, apoptosis was abrogated in miR-210transfected cells. These MSCs also expressed less caspase8. Since the MSCs were all from male rats, the authors ligated the left anterior descending coronary arteries of female Fischer 344 rats. The male MSCs were then injected around the infarcted area at multiple sites. The Sry gene is a sex-determining gene on the $\mathrm{Y}$ chromosome, which the investigators could then use to track their MSC. Caspase8 expression and TUNEL cells were reduced by the MSC treatment. The miR-210-MSC and control scramble-MSC both diminished infarct size and improved left ventricular fractional shortening, although the miR-210-harboring cells were somewhat better. We do not really know how the MSCs work. The idea that MSCs replace existing cardiomyocytes by "merely making more cardiomyocytes" is not
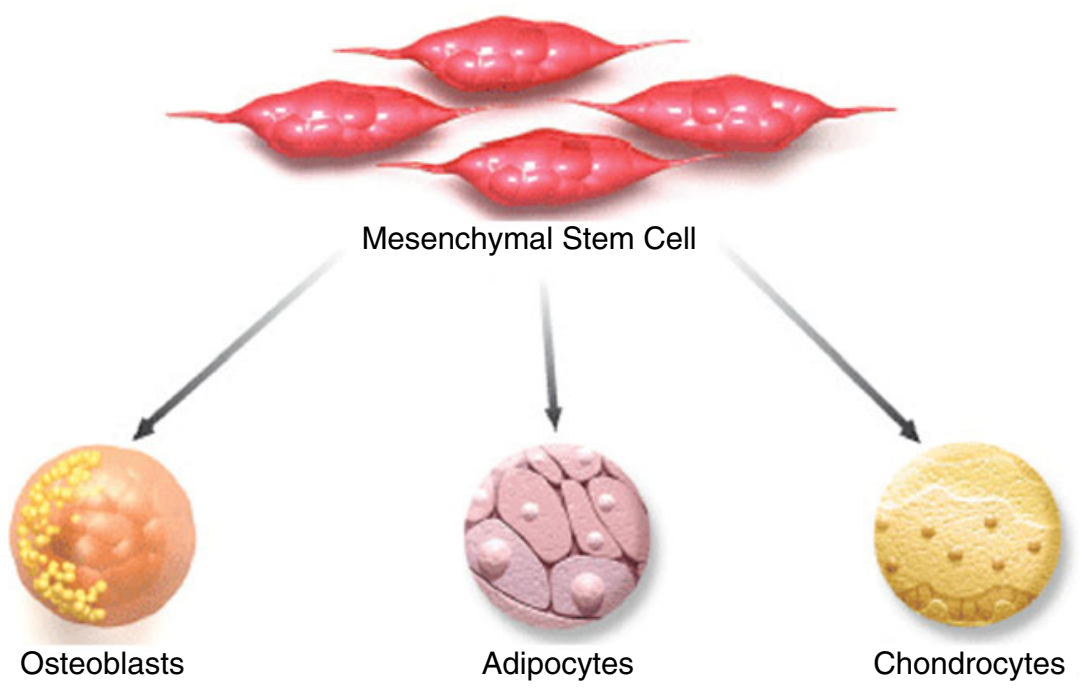

Mesenchymal stem-cell pathway 
Fig. 3 Gap junction channels, formed by connexin monomers such as connexin- 43 , facilitate the intercellular exchange of signaling molecules

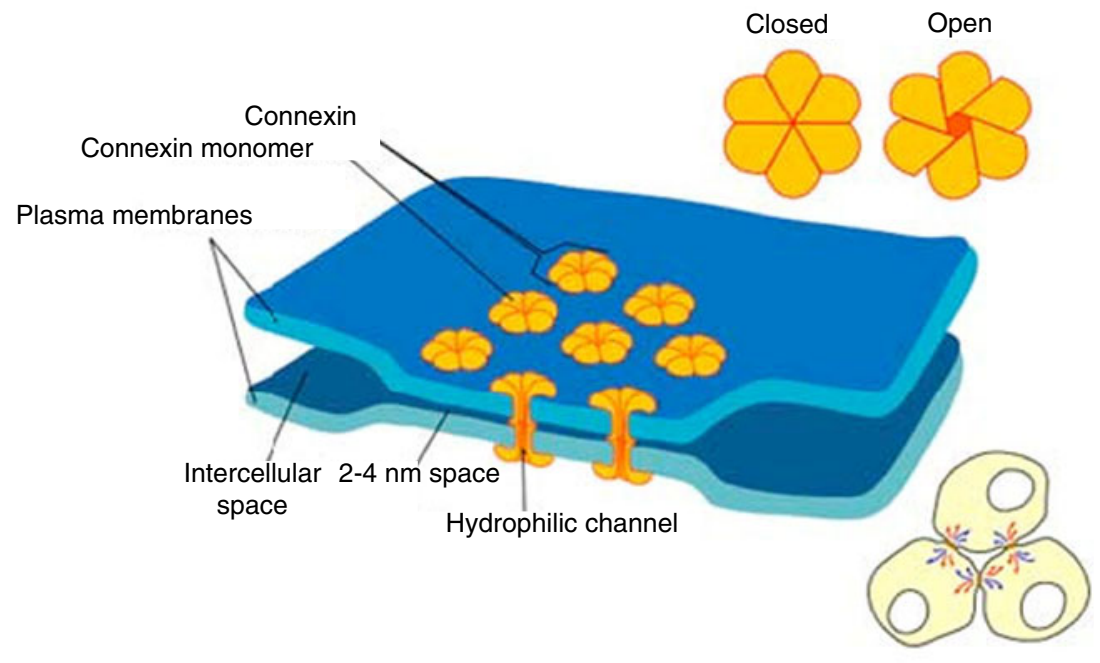

Gap Junction channels, formed by connexin monomers such as connexin-43, facilitate the intercellular exchange of signaling molecules substantiated scientifically. Thus, the function of MSC remains somewhat unclear. Heptanol is a seven-carbon alcohol that blocks gap junction channels (Fig. 3). Heptanol treatment abrogated the effects of MSC irrespective of the transfection, presumably by interfering with the transfer of miR-201 from transfected MSC to endogenous cardiomyocytes. The authors also show evidence from proliferation and endothelial markers that capillary and arteriolar density was improved by the treatment. Cell-transplanted heart sections were examined showing miR-210 localization in cardiomyocytes, but not in cell-transplanted heart sections from animals receiving the scrambled transfection constructs. Finally, co-culture experiments are presented showing connexin-43, the putative gap junction transfer mechanism for miR-210. Connexin-43 can indeed be blocked by heptanol.

Your editorialist, who is not an expert peer reviewer for this area, was impressed by the possibilities this study raised. First, miRs are a Nobel prize-winning discovery that offers a partial explanation of how we differ from our mouse friends who largely have the same structural genes that we have, but are different from us in many ways. The therapeutic targets provided by miR equal their numbers. Second, stem cells are coming and the strategy of using the recipients' own cells if possible solves an entire litany of problems. Finally, the idea of combining these strategies is a "tour-de-force." The current work has imperfections, including technical weaknesses. However, it scratches the surface of a very large "itch" from which we "gene therapy" buffs all suffer.

Respectfully,

Friedrich C. Luft
Acknowledgments FCL thanks the generous colleagues who placed these excellent illustrations into the Internet.

\section{References}

1. Zhu H, Fan G-C (2011) Role of microRNAs in the reperfused myocardium towards post-infarct remodeling. Cardiovasc Res 94:284-292

2. Huang X, Le Q-T, Giaccia AJ (2010) MiR-210 - micromanager of the hypoxia pathway. Trends Mol Med 16:230-237

3. Chan YC, Banerjee J, Choi SY, Sen CK (2012) miR-210: the master hypoxamir. Microcirculation 19:215-223

4. Keeting A (2012) Mesenchymal stromal cells: new directions. Cell Stem Cell 10:709-716

5. Tan J, Wu W, Xu X, Liao L, Zheng F, Messinger S, Sun X, Chen J, Yang S, Cai J et al (2012) Induction therapy with autologous mesenchymal stem cells in living-related kidney transplants: a randomized controlled trial. JAMA 307:11691177

6. Hare JM, Traverse JH, Henry TD, Dib N, Strumpf RK, Schulman SP, Gerstenblith G, DeMaria AN, Denktas AE, Gammon RS et al (2009) A randomized, double-blind, placebo-controlled, doseescalation study of intravenous adult human mesenchymal stem cells (prochymal) after acute myocardial infarction. J Am Coll Cardiol 54:2277-2286

7. Bolli R, Chugh AR, D'Amario D, Loughran JH, Stoddard MF, Ikram S, Beache GM, Wagner SG, Leri A, Hosoda T et al (2011) Cardiac stem cells in patients with ischaemic cardiomyopathy (SCIPIO): initial results of a randomised phase 1 trial. Lancet 378:1847-1857

8. Makkar RR, Smith RR, Cheng K, Malliaras K, Thomson LE, Berman D, Czer LS, Marbán L, Mendizabal A, Johnston PV et al (2012) Intracoronary cardiosphere-derived cells for heart regeneration after myocardial infarction (CADUCEUS): a prospective, randomised phase 1 trial. Lancet 379:895-904

9. Kim HW, Jiang S, Ashraf M, Haider KH (2012) Stem-cell based delivery of hypoxamir-210 to the infarcted heart: implications on stem-cell survival and preservation of infarcted heart function. $\mathrm{J}$ Mol Med. doi:10.1007/s00109-012-0920-1 